\title{
Gestão do cuidado em Odontologia: limites e potencialidades das ações na Estratégia Saúde da Família
}

\author{
Hellaynne Cláudia Heiko Yoshi Okuyama*; Rinaldo Henrique Aguilar-da-Silva** \\ * Mestre, Faculdade de Medicina de Marília (Famema), \\ Programa de Mestrado Profissional Ensino em Saúde \\ ** Professor Doutor, Faculdade de Medicina de Marília \\ (Famema), Programa de Mestrado Profissional Ensino em \\ Saúde
}

Recebido em 31/08/2017. Aprovado em 17/11/2017.

\begin{abstract}
RESUMO
O estudo objetivou avaliar os limites e as potencialidades das ações dos cirurgiões-dentistas (CD) sob a ótica da gestão do cuidado. A metodologia consistiu em uma abordagem quantiqualitativa, por meio de questionário destinado aos $\mathrm{CD}$ e entrevistas aos gestores municipais. Foram realizadas análise de conteúdo, na modalidade temática, e análise triangulada considerando o olhar dos $\mathrm{CD}$, gestores e o referencial bibliográfico. Os resultados apontaram a importância de conhecer o diagnóstico local, as necessidades de saúde e as prioridades trabalhadas. Limites e potencialidades foram identificados, e a transformação da prática foi considerada um desafio.
\end{abstract}

Descritores: Estratégia Saúde da Família. Odontologia. Saúde bucal. Sistema Único de Saúde. Gestão em Saúde.

\section{INTRODUÇÃO}

A Estratégia Saúde da Família (ESF) visa à reorganização da atenção básica de acordo com os princípios do Sistema Único de Saúde (SUS), fortalece o princípio da integralidade, a qualificação do acesso e do acolhimento com reorientação do processo de trabalho. Amplia a resolutividade da atenção à saúde com ênfase nas ações educativas e intersetoriais e no estabelecimento do vínculo entre usuários e equipe multiprofissional. Prioriza a longitu- dinalidade do cuidado, por meio do planejamento local com base no conhecimento do território, na identificação dos problemas de saúde e situações de risco da comunidade ${ }^{1}$.

A gestão do cuidado é o provimento ou a disponibilização das tecnologias de saúde, conforme as necessidades de cada indivíduo, em diferentes momentos, com o objetivo de promover segurança e autonomia, visando uma vida saudável e produtiva. A gestão do cuidado em saúde pode ser realizada nas dimensões 
individual, familiar, profissional, organizacional, sistêmica e societária, que se relacionam e apresentam interdependência, sendo representadas por atores ou protagonistas e determinados elementos ou lógica da dimensão ${ }^{2}$.

Além de superar a fragmentação e garantir a integralidade, a gestão do cuidado é representada por políticas, gestores, trabalhadores da saúde e usuários, que se articulam em diferentes espaços para resolver os problemas de saúde, utilizando a tecnologia mais adequada, no lugar e no tempo certo, para produção do cuidado. Para tanto, deve apoiar práticas individuais e coletivas com a continuidade do cuidado, possibilitando a construção de vínculos de confiança e autonomia ${ }^{3}$.

O cuidado ultrapassa a oferta de ações e de serviços, considera os sujeitos, suas vidas e as diferentes ações em saúde produzidas singularmente. Assim, além de institucional e físico, apresenta a dimensão afetiva com suas especificidades. Cuidar nem sempre significa agir, elaborar ações, executar procedimentos. Em alguns momentos, o sujeito necessita apenas exercer uma autonomia singular nesse território afetivo ${ }^{4}$.

Para a organização do modelo, o princípio da integralidade exige mudanças na formação e nas atitudes dos profissionais, para que as necessidades do indivíduo sejam vistas de uma forma integral ${ }^{5}$.

Considerar a complexidade da gestão do cuidado exige refletir e buscar estratégias para que a integralidade e a resolutividade das ações ocorram. Portanto, o investimento nos profissionais, sua aproximação com a realidade do território, ações efetivas de educação em saúde e organização do processo de trabalho são fatores essenciais.

Partindo do pressuposto de que há limites e potencialidades tanto na formação quanto na prática dos cirurgiões-dentistas em relação às ações desenvolvidas na ESF em busca de uma assistência baseada na integralidade e na gestão do cuidado, este estudo objetiva verificar os limites e as potencialidades identificados por esses profissionais e pela gestão municipal na realização das ações em saúde realizadas com foco nas necessidades de saúde da população, evidenciando como ocorre a gestão do cuidado em Odontologia.

\section{MÉTODO}

Trata-se de um estudo de campo, descritivo e com abordagem quantitativa e qualitativa. Os participantes deste estudo foram 27 cirurgiõesdentistas (CD) da Estratégia de Saúde da Família (ESF) do Município de Marília, sete apoiadores institucionais da Atenção Básica, um coordenador de assistência à saúde, um coordenador da Saúde Bucal e o Secretário Municipal da Saúde.

Por tratar-se de pesquisa com seres humanos o projeto foi submetido ao Conselho Municipal de Avaliação em Pesquisa (COMAP) da Secretaria Municipal de Saúde do município de Marília, protocolado sob o $\mathrm{n}^{\circ}$ 095/15-SS e pelo Comitê de Ética em Pesquisa da Famema, sendo aprovado com o Parecer $n^{\circ}$ 1.604.266.

A cada participante da pesquisa foi solicitada a assinatura do Termo de Consentimento Livre e Esclarecido e assegurada à liberdade de participação, de recusa e o direito de retirar-se da pesquisa a qualquer momento, sem que isso lhe causasse prejuízos ou constrangimentos.

A coleta de dados foi realizada em dois momentos, sendo que o primeiro se constituiu da aplicação de um questionário, aos $\mathrm{CD}$, com 20 assertivas referentes à gestão do cuidado, de acordo com a escala do tipo Likert, em que os respondentes emitem seu grau de concordância, por meio de alternativas (CF - Concordo Fortemente, C Concordo, N - Neutro, D - Discordo e DF - Discordo Fortemente). O segundo, constou de entrevistas semiestruturadas e áudio gravadas aos gestores municipais, orientadas pelo tema da atenção de saúde bucal no município.

Após a coleta, os dados quantitativos foram digitados em arquivos do Microsoft Office Excel (2010) e Word (2010) e calculados os percentuais 
das respostas, ilustradas por gráficos. Os dados qualitativos das entrevistas foram transcritos e analisados, segundo Bardin $^{6}$. Finalmente, a análise triangulada dos resultados considerou os dados quantitativos, qualitativos e o referencial bibliográfico, segundo Minayo et al. ${ }^{7}$. Para garantia do anonimato, os gestores foram identificados pela letra $\mathrm{G}$, seguida de números.

\section{RESULTADOS}

Os percentuais dos resultados quantitativos estão representados na tabela 1 . A análise revelou que a organização do processo de trabalho atual na Unidade de Saúde da Família (USF) não supre a necessidade do profissional, da população e da gestão municipal. Esses resultados apontam que o processo de trabalho necessita ser reavaliado para que os fatores que interferem nos resultados alcançados e na qualidade do cuidado sejam considerados.

As entrevistas realizadas com os gestores foram orientadas pelo tema da atenção de saúde bucal no município, incluindo pressupostos da ESF, existência de limites ou potencialidades para a realização das ações, com ênfase na integralidade do cuidado, considerando as necessidades e a realidade da população local para o planejamento das ações pelas Equipes de Saúde Bucal.

Tabela 1 - Respostas ao questionário sobre Processo de Trabalho

\begin{tabular}{|c|c|c|c|c|c|}
\hline \multirow[b]{2}{*}{ QUESTÕES } & \multicolumn{5}{|c|}{ ALTERNATIVAS } \\
\hline & $\begin{array}{c}\text { (CF) } \\
\mathrm{n} \\
(\%)\end{array}$ & $\begin{array}{l}2(\mathbf{C}) \\
\mathrm{n} \\
(\%)\end{array}$ & $\begin{array}{c}3(\mathbf{N}) \\
\mathrm{n} \\
(\%)\end{array}$ & $\begin{array}{c}4(\mathrm{D}) \\
\mathrm{n} \\
(\%)\end{array}$ & \begin{tabular}{|c}
$\mathbf{5}$ (DF) \\
$\mathrm{n}$ \\
$(\%)$
\end{tabular} \\
\hline $\begin{array}{l}\text { Q1. Os cirurgiões-dentistas atuam diariamente na gestão local } \\
\text { da unidade, participam das discussões de assuntos diversos e } \\
\text { responsabilizam-se pelas decisões junto à equipe. }\end{array}$ & $\begin{array}{c}18 \\
66,66\end{array}$ & $\begin{array}{c}08 \\
29,63\end{array}$ & $\begin{array}{c}00 \\
0,00\end{array}$ & $\begin{array}{c}01 \\
3,70\end{array}$ & $\begin{array}{c}00 \\
0,00\end{array}$ \\
\hline $\begin{array}{l}\text { Q2. Há resistência dos integrantes da equipe e da comunidade } \\
\text { em relação à inserção do CD nas atividades propostas pela } \\
\text { ESF, como grupos, gestão local, visitas domiciliares, } \\
\text { limitando a efetivação do processo de trabalho. }\end{array}$ & $\begin{array}{c}00 \\
0,00\end{array}$ & $\begin{array}{c}04 \\
14,81\end{array}$ & $\begin{array}{c}01 \\
3,70\end{array}$ & $\begin{array}{c}16 \\
59,26\end{array}$ & $\begin{array}{c}06 \\
22,22\end{array}$ \\
\hline $\begin{array}{l}\text { Q3. A formação na graduação, considerando a organização } \\
\text { curricular e a metodologia de ensino aprendizagem, influi no } \\
\text { processo de trabalho, dificultando a integração com os demais } \\
\text { membros da equipe. }\end{array}$ & $\begin{array}{c}02 \\
7,40\end{array}$ & $\begin{array}{c}08 \\
29,63\end{array}$ & $\begin{array}{c}03 \\
11,11\end{array}$ & $\begin{array}{c}13 \\
48,15\end{array}$ & $\begin{array}{c}01 \\
3,70\end{array}$ \\
\hline $\begin{array}{l}\text { Q4. Para a adequação do processo de trabalho em saúde bucal, } \\
\text { é necessário considerar a interdisciplinaridade e multipro- } \\
\text { fissionalismo. }\end{array}$ & $\begin{array}{c}09 \\
33,33\end{array}$ & $\begin{array}{c}16 \\
59,26\end{array}$ & $\begin{array}{c}02 \\
7,40\end{array}$ & $\begin{array}{c}00 \\
0,00\end{array}$ & $\begin{array}{c}00 \\
0,00\end{array}$ \\
\hline $\begin{array}{l}\text { Q5. Integralidade da atenção, intersetorialidade, ampliação e } \\
\text { qualificação da assistência e, ainda, condições de trabalho } \\
\text { colaboram na adequação do processo de trabalho. }\end{array}$ & $\begin{array}{c}11 \\
40,74\end{array}$ & $\begin{array}{c}15 \\
55,55\end{array}$ & $\begin{array}{c}01 \\
3,70\end{array}$ & $\begin{array}{c}00 \\
0,00\end{array}$ & $\begin{array}{c}00 \\
0,00\end{array}$ \\
\hline $\begin{array}{l}\text { Q6. A comunicação entre os setores relacionados ao processo } \\
\text { de trabalho odontológico ocorre de forma satisfatória, não } \\
\text { influenciando o resultado do atendimento. }\end{array}$ & $\begin{array}{c}00 \\
0,00\end{array}$ & $\begin{array}{c}09 \\
33,33\end{array}$ & $\begin{array}{c}03 \\
11,11\end{array}$ & $\begin{array}{c}15 \\
55,55\end{array}$ & $\begin{array}{c}00 \\
0,00\end{array}$ \\
\hline $\begin{array}{l}\text { Q7. A organização do processo de trabalho atual na unidade } \\
\text { de saúde da família supre as necessidades do profissional, da } \\
\text { população e da gestão municipal. }\end{array}$ & $\begin{array}{c}00 \\
0,00\end{array}$ & $\begin{array}{c}05 \\
18,52\end{array}$ & $\begin{array}{c}04 \\
14,81\end{array}$ & $\begin{array}{c}15 \\
55,55\end{array}$ & $\begin{array}{c}03 \\
11,11\end{array}$ \\
\hline
\end{tabular}




\begin{tabular}{|c|c|c|c|c|c|}
\hline $\begin{array}{l}\text { Q8. Diante do cadastramento das famílias, do reconhecimento } \\
\text { da área e de reuniões com a comunidade, uma estratégia é a } \\
\text { capacitação em saúde bucal dos agentes comunitários de saúde } \\
\text { para que sejam multiplicadores. }\end{array}$ & $\begin{array}{c}09 \\
33,33\end{array}$ & $\begin{array}{c}17 \\
62,96\end{array}$ & $\begin{array}{c}01 \\
3,70\end{array}$ & $\begin{array}{c}00 \\
0,00\end{array}$ & $\begin{array}{c}00 \\
0,00\end{array}$ \\
\hline $\begin{array}{l}\text { Q9. O planejamento local e a programação das ações } \\
\text { dependem do envolvimento dos sujeitos, da determinação e do } \\
\text { comprometimento em realizar mudança. }\end{array}$ & $\begin{array}{c}14 \\
51,85\end{array}$ & $\begin{array}{c}12 \\
44,44\end{array}$ & $\begin{array}{c}01 \\
3,70\end{array}$ & $\begin{array}{c}00 \\
0,00\end{array}$ & $\begin{array}{c}00 \\
0,00\end{array}$ \\
\hline $\begin{array}{l}\text { Q10. Planejar implica definir objetivos, metas e ações que } \\
\text { serão realizadas para o enfrentamento das prioridades, } \\
\text { estabelecendo responsáveis, prazos e recursos envolvidos. }\end{array}$ & $\begin{array}{c}15 \\
55,55\end{array}$ & $\begin{array}{c}11 \\
40,74\end{array}$ & $\begin{array}{c}01 \\
3,70\end{array}$ & $\begin{array}{c}00 \\
0,00\end{array}$ & $\begin{array}{c}00 \\
0,00\end{array}$ \\
\hline $\begin{array}{l}\text { Q11. A epidemiologia e as informações sobre o território devem } \\
\text { ser utilizadas para o planejamento das ações, mediante um } \\
\text { diagnóstico das doenças prevalentes, condições socioeconômicas, } \\
\text { hábitos, estilos de vida e necessidades de saúde. }\end{array}$ & $\begin{array}{c}19 \\
70,37\end{array}$ & $\begin{array}{c}08 \\
29,63\end{array}$ & $\begin{array}{c}00 \\
0,00\end{array}$ & $\begin{array}{c}00 \\
0,00\end{array}$ & $\begin{array}{c}00 \\
0,00\end{array}$ \\
\hline $\begin{array}{l}\text { Q12. As reuniões de apoio representam espaços de avaliação } \\
\text { do processo de trabalho em que as equipes podem trocar } \\
\text { experiências e discutir problemas comuns. }\end{array}$ & $\begin{array}{c}08 \\
29,63\end{array}$ & $\begin{array}{c}14 \\
51,85\end{array}$ & $\begin{array}{c}01 \\
3,70\end{array}$ & $\begin{array}{c}02 \\
7,40\end{array}$ & $\begin{array}{c}02 \\
7,40\end{array}$ \\
\hline $\begin{array}{l}\text { Q13. O processo de Educação Permanente e Continuada é } \\
\text { fundamental para promover o trabalho na ESF com atualização } \\
\text { de conceitos, motivação e valorização profissional. }\end{array}$ & $\begin{array}{c}18 \\
66,66\end{array}$ & $\begin{array}{c}09 \\
33,33\end{array}$ & $\begin{array}{c}00 \\
0,00\end{array}$ & $\begin{array}{c}00 \\
0,00\end{array}$ & $\begin{array}{c}00 \\
0,00\end{array}$ \\
\hline $\begin{array}{l}\text { Q14. O enfoque de risco para identificar as populações com } \\
\text { maior vulnerabilidade, estabelecendo prioridades na atenção à } \\
\text { saúde, é pouco utilizado. }\end{array}$ & $\begin{array}{c}04 \\
14,81\end{array}$ & $\begin{array}{c}16 \\
59,26\end{array}$ & $\begin{array}{c}02 \\
7,40\end{array}$ & $\begin{array}{c}04 \\
14,81\end{array}$ & $\begin{array}{c}01 \\
3,70\end{array}$ \\
\hline $\begin{array}{l}\text { Q15. Conflitos interpessoais interferem no funcionamento da } \\
\text { unidade e dificultam a implementação do programa. }\end{array}$ & $\begin{array}{c}13 \\
48,15 \\
\end{array}$ & $\begin{array}{c}13 \\
48,15 \\
\end{array}$ & $\begin{array}{c}01 \\
3,70 \\
\end{array}$ & $\begin{array}{c}00 \\
0,00 \\
\end{array}$ & $\begin{array}{c}00 \\
0,00 \\
\end{array}$ \\
\hline $\begin{array}{l}\text { Q16. É necessária uma reflexão sobre educação em saúde, } \\
\text { utilizando-a em grupos com o enfoque da saúde coletiva, } \\
\text { levando a participação dos indivíduos na melhoria das } \\
\text { condições de vida e saúde de forma mais compartilhada. }\end{array}$ & $\begin{array}{c}08 \\
29,63\end{array}$ & $\begin{array}{c}18 \\
66,66\end{array}$ & $\begin{array}{c}01 \\
3,70\end{array}$ & $\begin{array}{c}00 \\
0,00\end{array}$ & $\begin{array}{c}00 \\
0,00\end{array}$ \\
\hline $\begin{array}{l}\text { Q17. Cada equipe define como organizar as ações, como } \\
\text { agendamento de consultas, seleção de usuários e periodicidade } \\
\text { de atividades. }\end{array}$ & $\begin{array}{c}07 \\
25,93\end{array}$ & $\begin{array}{c}19 \\
70,37\end{array}$ & $\begin{array}{c}01 \\
3,70\end{array}$ & $\begin{array}{c}00 \\
0,00\end{array}$ & $\begin{array}{c}00 \\
0,00\end{array}$ \\
\hline $\begin{array}{l}\text { Q18. O auxiliar de saúde bucal centra suas ações em atividades } \\
\text { convencionais, como instrumentação, desinfecção e } \\
\text { esterilização. }\end{array}$ & $\begin{array}{c}03 \\
11,11\end{array}$ & $\begin{array}{c}08 \\
29,63\end{array}$ & $\begin{array}{c}01 \\
3,70\end{array}$ & $\begin{array}{c}12 \\
44,44\end{array}$ & $\begin{array}{c}03 \\
11,11\end{array}$ \\
\hline $\begin{array}{l}\text { Q19. A equipe interdisciplinar é uma potencial ferramenta de } \\
\text { mudança nos processos de trabalho, fortalecendo } \\
\text { integralidade, acolhimento, } \\
\text { resolubilidade e responsabilização. }\end{array}$ & $\begin{array}{c}20 \\
74,07\end{array}$ & $\begin{array}{c}06 \\
22,22\end{array}$ & $\begin{array}{c}00 \\
0,00\end{array}$ & $\begin{array}{c}01 \\
3,70\end{array}$ & $\begin{array}{c}00 \\
0,00\end{array}$ \\
\hline $\begin{array}{l}\text { Q20. Há falta de formação em equipe multiprofissional no } \\
\text { trabalho e deficiência no conhecimento das atribuições pelos } \\
\text { membros da ESF. }\end{array}$ & $\begin{array}{c}06 \\
22,22\end{array}$ & $\begin{array}{c}16 \\
59,26\end{array}$ & $\begin{array}{c}02 \\
7,40\end{array}$ & $\begin{array}{c}03 \\
11,11\end{array}$ & $\begin{array}{c}00 \\
0,00\end{array}$ \\
\hline
\end{tabular}

CF: Concordo Fortemente C: Concordo N: Neutro $\quad$ D: Discordo DF: Discordo Fortemente 
A análise temática das entrevistas identificou como Núcleo de Sentido o Processo de Trabalho das equipes de saúde bucal. Assim, o produto das unidades de contexto e de registro possibilitaram a estruturação em 4 categorias: I) Cirurgião-dentista na gestão local da unidade, II) Trabalho em equipe multiprofissional, III) Vínculo, corresponsabilização e autonomia do cuidado e IV) Cuidado ampliado.

\section{DISCUSSÃO}

As Diretrizes Curriculares Nacionais para o Curso de Graduação em Odontologia especificam a importância do CD participar do gerenciamento, exercer a administração dos recursos físicos, materiais e de informação, atuar como empreendedor, gestor, empregador ou exercer liderança na equipe de saúde sendo capaz de aprender continuamente na sua formação e na sua prática ${ }^{8}$.

Os dados quantitativos para a categoria cirurgião-dentista na gestão local da unidade demonstram que a grande maioria $(96,29 \%)$ concordou fortemente ou concordou com a assertiva sobre atuação diária, participação das discussões e corresponsabilização pelo território.

Isso foi corroborado pelos discursos dos gestores que revelaram a importância do odontólogo na gestão da unidade. Esses consideraram como primordial o olhar diferenciado do $\mathrm{CD}$ e sua participação na organização do processo de trabalho de toda a equipe. Ressaltaram ainda a necessidade de envolvimento, perfil, tempo disponível para a gestão, além da organização do trabalho e reflexão sobre a prática:

" $O$ dentista [...], curricularmente, não tem a questão de gestão de serviço [...], precisa casar com o perfil. Então se é um profissional que gosta da gestão, ele deslancha, ele consegue opinar, trabalhar em equipe, organizar o serviço não só da odontologia [...]”.'. (G2)
Para que a gestão do cuidado se efetive é necessário, ainda, realizar o planejamento e programação das ações, o que para 96,29\% dos CD implica definir objetivos, metas e ações para o enfrentamento de prioridades, identificando responsáveis, prazos e recursos necessários. Em nosso estudo houve uma concordância tanto sob o olhar dos CD quanto dos gestores, sobre o fato de o planejamento das ações sofrer influências do envolvimento e do comprometimento dos sujeitos. Apesar de os profissionais revelarem conhecimento sobre o assunto, na opinião dos gestores, as equipes desenvolvem o trabalho sem planejamento adequado, provavelmente devido à fragilidade de se trabalhar com indicadores de saúde, ao grande volume de atribuições e à rotina atribulada por demandas diversas.

Assim, o planejamento precisa estar integrado às necessidades em saúde, com ações intersetoriais necessárias para o cuidado ampliado 9 .

Sobre o Trabalho em equipe multiprofissional, a maioria dos profissionais discordou com a assertiva referente à resistência dos integrantes da equipe e da comunidade em relação à inserção do $\mathrm{CD}$ nas atividades propostas pela ESF, como grupos, gestão local, visitas domiciliares, limitando a efetivação do processo de trabalho, diferente do achado de Costa et al. ${ }^{10}$, em que os CD não participaram de forma integrada na equipe, bem como a articulação das ações entre os diversos profissionais não ocorria de forma sistemática, como parte do processo de trabalho.

Diferente da maioria das realidades, nas 35 USF do Município de Marília, a gestão local é de responsabilidade do chamado trio gestor, constituído por enfermeiro, médico e cirurgiãodentista. Essa configuração permite que o $\mathrm{CD}$ não fique centrado no atendimento odontológico, mas participe efetivamente das decisões e responsabilidades e ocorra sua integração na equipe: 
"[...] o dentista fazer parte do trio gestor é uma coisa importante porque ele dá sua opinião, [...] ele acaba fazendo gestão, que é importantíssimo.[...]. " (G6)

Entretanto, apesar da atuação na gestão local, $81,48 \%$ dos CD concordam que existe falta de formação no trabalho em equipe multiprofissional e deficiência nas atribuições dos membros da equipe. Isso mostrou estar em consonância com a opinião dos gestores que consideraram o trabalho em equipe um potencial, porém relataram existir falta de conhecimento dos profissionais sobre as atribuições dos membros da ESF, em convergência com o estudo realizado por Moretti-Pires et al. ${ }^{11}$. Isso pode ser um indicativo de que conhecer deve ir além da mera descrição das competências comuns e específicas dos profissionais que compõem a equipe. Significa estar disposto a vivenciar as competências colaborativas bem como exercitar a resiliência necessária para o trabalho multiprofissional e efetiva colaboração, como fatores essenciais para o bom desempenho e resolutividade das ações na saúde pública.

Considerando isso, o trabalho multidisciplinar apresenta-se como um requisito para a atuação na ESF. Nesse estudo, observamos quantitativamente a concordância quase absoluta $(96,29 \%)$ sobre a potencialidade da equipe como ferramenta de mudanças no processo de trabalho. Santos e Assis ${ }^{12}$ destacam que o mesmo fortalece a integralidade, o acolhimento, o vínculo, a autonomia e responsabilização. Assim, a questão que se subjaz é: se existe o reconhecimento dos CD acerca da importância e benefícios do trabalho multidisciplinar por que encontramos dificuldades em efetivá-lo? Nossa percepção aponta para uma construção histórica da função do odontólogo com atividades assistenciais restritas e isoladas revelando o desafio de reconstruir junto à população adscrita e com a própria equipe uma nova maneira de ser e agir no processo de trabalho.
Outro aspecto que chamou atenção nos relatos dos gestores referiu-se a rotatividade dos profissionais como um fator complicador para a formação do vínculo. Essa condição, sempre que presente exige o retrabalho, o recomeço dessa construção de laços de confiança, reforçando os achados de Padula e Aguilar-da-Silva13. Certamente, a permanência do $\mathrm{CD}$ atuando em uma mesma equipe, se apropriando das necessidades de saúde da população e desenvolvendo ações que vem ao encontro dessas necessidades, possibilita a qualificação do trabalho. Por outro lado, há situações que favorecem essa rotatividade, como o próprio desejo do CD em mudar de unidade, na maioria das vezes justificados pela localização geográfica, pelos relacionamentos interpessoais entre os membros da equipe, e principalmente, se o odontólogo não supre satisfatoriamente as necessidades esperadas.

"[...] essa grande rotatividade que às vezes a gente tem, a hora que o dentista consegue começar fazer um trabalho, às vezes tem essa troca, [...], mas aí, começar tudo de novo, pegar no meio do caminho, [...], isso quebra muito o vínculo". (G6)

Segundo Pimentel et al. ${ }^{14}$ conciliar e integrar as ações dos diferentes profissionais das USFs não é uma tarefa fácil, pois nem sempre os mesmos estão preparados e com disposição para agir de forma integrada. Apesar disso, ressaltaram que muitas equipes de saúde bucal referiram ter uma boa integração com a ESF. Interessantemente encontramos resultados similares que revelaram a participação dos CD no planejamento das ações e em campanhas e grupos educativos apontando que o atendimento qualificado, resolutivo e integral ao usuário é o principal objetivo do trabalho no SUS.

Apesar da importância do trabalho em equipe, segundo Moraes et al. ${ }^{15}$ algumas equipes de saúde bucal não participam de reuniões, 
reforçando a sensação de não fazerem parte da equipe multiprofissional. Este resultado se contrapõe à realidade encontrada no município em estudo, pois $81,48 \%$ dos participantes referiram não haver resistência da equipe nem da comunidade sobre a inserção do $\mathrm{CD}$ nas atividades propostas pela ESF. Do ponto de vista qualitativo, os gestores reconheceram a importância da atuação do $\mathrm{CD}$ na equipe multiprofissional, assim como o estudo de Soares et al. ${ }^{16}$.

$O$ Vínculo, a corresponsabilização e a autonomia do cuidado são elementos essenciais para a adesão dos usuários ao serviço de saúde, pois sentem-se seguros, ouvidos e alvo de cuidados. Esses fatores presentes nas práticas cotidianas entre profissionais e população, acompanhados por diálogo e respeito, revelando ir o cuidado além da dimensão biológica da doença ${ }^{17}$.

Os relatos dos gestores reforçaram que a atuação da equipe de saúde bucal na ESF possibilita a aproximação com a família, não se restringe ao tratamento individual e permite o estabelecimento de vínculo entre profissionais e usuários. Os entrevistados reconheceram, porém, a existência de grande limitação para o profissional conseguir, de fato, que a população assuma a corresponsabilidade pelo cuidado integral. Já sob o ponto de vista quantitativo, a maioria dos $\mathrm{CD}(77,78 \%)$ referiu que atua com a equipe, identifica e utiliza espaços sociais, o que fortalece o vínculo e a parceria com a população, conforme consta nas Diretrizes da Política Nacional de Saúde Bucal ${ }^{18}$.

"[...] quando você fala da integralidade, você fala também da correspon-sabilidade. Aí que eu acho difícil, em que momento, e de que forma a população faz o que the compete também, [...] de conseguir atingir um pouco mais do autocuidado, do sujeito corresponsável pela sua saúde de forma integral [...]". (G2)
A 'autonomia' possibilita que o sujeito seja protagonista do processo, assumindo corresponsabilidade nos resultados da ação. Para isso, faz-se necessário refletir e tomar consciência do paradigma de pensar e agir em saúde $^{19}$. Para os $C D$, a equipe é uma ferramenta potente que possibilita o fortalecimento da autonomia. Os relatos dos gestores demonstraram que o alcance da autonomia ainda representa um limite para as ações dos profissionais, já que depende da corresponsabilização do sujeito pelo seu autocuidado.

Há necessidade de reflexão sobre o real sentido da corresponsabilidade prevista pela ESF, considerando a dificuldade de tornar a população corresponsável de um programa que eles desconhecem ${ }^{20}$.

A relação entre profissionais e usuários é tema desafiador para a reorganização dos serviços de saúde pública. Imprescindível promover a autonomia dos usuários, a construção da promoção da saúde, superando a vulnerabilidade de quem cuida e de quem é cuidado, no processo de acolhimento que, por sua vez, torna a relação menos verticalizada, mais contextualizada e humanizada ${ }^{21}$.

Assim, em relação ao 'cuidado ampliado', observamos que $96,29 \%$ dos participantes concordaram que a integralidade da atenção, intersetorialidade, ampliação e qualificação da assistência e condições de trabalho colaboram na adequação do processo de trabalho.

Houve uma tendência à divergência nas respostas dos $\mathrm{CD}$ na assertiva que abordou a organização do processo de trabalho atual na USF ser capaz de suprir as necessidades do profissional, da população e da gestão municipal, uma vez que $66,66 \%$ dos profissionais discordaram da assertiva. Este dado sugere a importância de se refletir sobre a prática para possibilitar adequações nesta organização. Sob o ponto de vista qualitativo, os gestores revelaram 
que existem inúmeros limites referentes ao cuidado ampliado, como o envolvimento e o comprometimento dos profissionais, mudança de conceitos e necessidade de considerar as exigências reais desta população, com toda a complexidade e particularidades do território e reflexão sobre a prática:

"[...] pra considerar aquela comunidade que ele atende, na sua integralidade, fazendo prevenção não só das doenças da boca, [...] mas no geral, considerando a realidade daquela população, os equipamentos sociais [...]”. (G4)

Reorganizar as práticas das equipes da ESF implica redirecionar a atenção à saúde para a 'integralidade' e a equidade, a fim de que a atenção às famílias, em um dado território geossocial, considere também as necessidades em saúde dessas famílias. Os serviços devem estar organizados de forma a responder a essas necessidades e norteados pela concepção de saúde e doença capaz de interligar os aspectos individuais aos coletivos. Assim, os trabalhadores devem considerar que as necessidades, uma vez satisfeitas, serão reiteradas ou ampliadas, o que exigirá a instauração de um novo processo de trabalho para continuar a atender a elas ${ }^{22}$. A qualidade de vida e saúde depende de inúmeras necessidades, que abrangem diversas dimensões, imprescindíveis para a vida no seu sentido mais amplo, com aspectos individuais, emocionais, afetivos, sociais e humanos.

Sob o olhar qualitativo, os relatos dos gestores demonstram existir uma divergência entre o discurso dos profissionais e a prática realizada por eles, o que pode ser atribuído à própria necessidade de compreensão das propostas da ESF, bem como à importância de considerar as mudanças na saúde do País.

“[...] o momento que se encontra a saúde no Brasil, um momento de mudanças, de pensar, de reflexão, e de quebra de paradigmas que envolvem todos os níveis, não só a população, mas profissionais, gestão local, gestão Municipal, Estadual e até Federal". (G5)

Os gestores afirmam ainda que, embora tenham ocorrido inúmeras mudanças de conceitos sobre saúde e cuidado, para que, de fato, ocorram atitudes transformadoras no atendimento à população, com vistas ao cuidado ampliado, é necessário haver avanços na própria gestão. As ações devem ser desencadeadas, considerando a importância da reflexão e avaliação dos recursos disponíveis. Salienta-se, porém, que muitas ações não dependem necessariamente da gestão municipal ou de recursos materiais, já que cada equipe pode e deve refletir sobre a própria autonomia e suas possibilidades, utilizando tecnologias leves.

"[...] várias coisas mudaram em relação ao que se pensa sobre saúde, ao que se pensa sobre cuidado, e pra que essas reflexões, essas mudanças, e o olhar integral cheguem até o profissional que tá lá atendendo, que tá ali com o usuário, elas precisam começar na gestão, e ai ser um desencadeante". (G8)

A Atenção Primária se sente pressionada a cumprir metas definidas pelos indicadores de saúde e números de atendimentos, como se pudesse, de maneira isolada, ordenar e regular a integração entre os três níveis de atenção do SUS, evidenciando a dificuldade de integração das ações da unidade com os demais níveis de cuidado $^{23}$.

As equipes não conseguem trabalhar sozinhas todas as questões que influenciam e determinam a saúde e a doença das populações, sendo importante a valorização das tecnologias leves ${ }^{24}$, corroborando a opinião dos gestores entrevistados em nosso estudo.

O cuidado ampliado foi considerado um desafio para as equipes e para os gestores, dependendo da complexidade de fatores e dos 
atores envolvidos no processo.

\section{CONCLUSÕES}

Os resultados deste estudo revelaram que a gestão do cuidado representa um grande desafio, considerando a abordagem de forma integral, humanizada, com valorização dos profissionais, reconhecimento das necessidades da população e otimização de recursos e espaços disponíveis.

Em contrapartida, constatamos a existência de potencialidades que precisam ser reconhecidas e valorizadas, como por exemplo, o trabalho em equipe multiprofissional que fortalece o vínculo, a corresponsabilidade e a autonomia e, ainda, possibilita mudanças no processo de trabalho. Os gestores reconhecem o trabalho realizado pelos $\mathrm{CD}$, e ressaltam que a atuação do odontólogo na gestão local da unidade, apesar da influência do perfil do profissional, é considerada um diferencial no município.

Verificamos que existe confluência de entendimentos referente à gestão do cuidado para $\mathrm{CD}$ e gestores. Cabe ressaltar, porém, que os odontólogos reconhecem a importância dessa temática muitas vezes negligenciada durante a formação. Além disso, destaca-se a necessidade da continuidade da abordagem integral para atender as necessidades de saúde da população, e contemplar as habilidades e competências dos profissionais do SUS.

Inferimos que apesar do reconhecimento de potencialidades no trabalho realizado pelos cirurgiões-dentistas, existem limites e necessidades para ampliar o cuidado, para que mudanças efetivamente ocorram. Um fator limitante observado é que o enfoque de risco para o diagnóstico das necessidades de saúde da população e suas prioridades ainda é pouco utilizado e que a gestão do cuidado depende do envolvimento e do comprometimento dos sujeitos, incluindo usuários, profissionais e gestores.
Considerando a amplitude e a complexidade da integralidade do cuidado, sugerimos aprofundamento e continuidade de pesquisas referentes ao tema, para subsidiar o fortalecimento da gestão do cuidado em odontologia.

\section{ABSTRACT \\ Care management in Dentistry: limits and potentialities of actions in Family Health Strategy}

The purpose of this study was to evaluate the limits and potentialities of dental surgeons'(DS) actions under the view of care management. The methodology consisted of a quantiqualitative approach, through questionnaire destined to DS and interviews for the municipal managers. Content analyses were performed, in the theme modality, and triangulated analysis considering the view of DS, managers and bibliographical reference. The results have shown the importance of knowing the local diagnosis, the needs of health and priorities worked on. Limits and potentialities were identified, and the transformation of practice was considered a challenge.

Descriptors: Family Health Strategy. Dentistry. Oral Health. Unified Health System. Health Management.

\section{REFERÊNCIAS}

1. Brasil. Ministério da Saúde. Política Nacional de Atenção Básica. Brasília: Ministério da Saúde; 2012.

2. Cecilio LCO. Apontamentos teóricoconceituais sobre processos avaliativos considerando as múltiplas dimensões da gestão do cuidado em saúde. Interface. 2011;15(37):589-99.

3. Santos AM. Gestão do cuidado na microrregião de saúde de Vitória da Conquista (Bahia): desafios para constituição de rede regionalizada com cuidados coordenados pela Atenção Primária à Saúde [tese]. Rio de Janeiro: Escola Nacional de Saúde Pública Sergio 
Arouca; 2013. $332 \mathrm{p}$.

4. Silva MRF, Silveira LC, Pontes RJS, Vieira AN. O cuidado além da saúde: cartografia do vínculo, autonomia e território afetivo na saúde da família. REME Rev Min Enferm. 2015;19(1):249-54.

5. Albuquerque PC, Stotz EN. A educação popular na atenção básica à saúde no município: em busca da integralidade. Interface. 2004;8(15):259-74.

6. Bardin L. Análise de conteúdo. São Paulo: Edições 70; 2012.

7. Minayo MCS, Souza ER, Constantino P, Santos NC. Métodos, técnicas e relações em triangulação. In: Minayo MCS, Assis SG, Souza ER. Avaliação por triangulação de métodos: abordagem de programas sociais. Rio de Janeiro: Fiocruz; 2005. p. 71-103.

8. Brasil. Ministério da Educação. Conselho Nacional de Educação. Resolução CNE/CES n ${ }^{\circ}$ 3, de 19 de fevereiro de 2002. Dispõe sobre as Diretrizes Curriculares Nacionais do curso de graduação em Odontologia. Diário Oficial da União, Brasília (DF); 4 mar 2012; Seção 1:10.

9. Junges JR, Barbiani R, Zoboli ELCP. Planejamento estratégico como exigência ética para a equipe e a gestão local da atenção básica em saúde. Interface. 2015;19(53):265-74.

10. Costa RM, Medeiros Júnior AM, Céu Clara ÍC, Pinheiro IVA. O trabalho em equipe desenvolvido pelo cirurgião-dentista na Estratégia Saúde da Família: expectativas, desafios e precariedades. Rev Bras Med Fam Comun. 2012;7(24):147-63.

11. Moretti-Pires RO, Lima LAM, Machado MH. Sociologia das profissões e percepção de acadêmicos de Odontologia sobre o Agente Comunitário de Saúde em saúde bucal. Interface. 2011;15(39):1085-96.

12. Santos A, Assis M. Da fragmentação à integralidade: construindo (des)construindo a prática de saúde bucal no Programa de Saúde da Família (PSF) de Alagoinhas, BA. Ciênc Saúde Coletiva. 2006;11(1):53-61.

13. Padula MGC, Aguilar-da-Silva RH . Professional profile of dentists who are members of the Family Health Strategy city of Marília, São Paulo: the challenge of interprofessional work. Rev Odontol UNESP. 2014;43(1):52-60.

14. Pimentel FC, Albuquerque PC, Martelli PJL, Souza WV, Acioli RML. Caracterização do processo de trabalho das equipes de saúde bucal em municípios de Pernambuco, Brasil, segundo porte populacional: da articulação comunitária à organização do atendimento clínico. Cad Saúde Pública. 2012;28 (Suppl):146-57.

15. Moraes LB, Kligerman DC, Cohen SC. Análise do perfil sociodemográfico e do processo de trabalho do cirurgião-dentista inserido no Programa de Saúde da Família em três municípios da região serrana do Estado do Rio de Janeiro. Physis. 2015;25(1):171-86.

16. Soares ÉF, Reis SCGB, Freire MCM. Percepção dos trabalhadores da Estratégia Saúde da Família sobre a atuação das equipes de saúde bucal em Goiânia, em 2009: estudo qualitativo. Epidemiol Serv Saúde. 2013;22(3):483-90.

17. Santos AM, Nóbrega IKS, Assis MMA, Jesus SR, Kochergin CN, Bispo Júnior JP, Alves JS, Santana KC. Desafios à gestão do trabalho e educação permanente em saúde para a produção do cuidado na estratégia saúde da família. Rev APS. 2015;18(1):3949.

18. Brasil. Ministério da Saúde. Diretrizes da Política Nacional de Saúde Bucal. Brasília: Ministério da Saúde; 2004.

19. Souza MF. A gestão do cuidado odontológico no Programa Saúde da Família 
no Centro de Saúde Goiânia - Belo Horizonte [monografia na Internet]. Belo Horizonte: Universidade Federal de Minas Gerais; 2010. 33 p. [Acesso em 28 maio 2016]. Disponível em: https://www.nescon. medicina.ufmg.br/biblioteca/imagem/2831. pdf

20. Gelinski CROG. A questão da coresponsabilidade prevista na Estratégia Saúde da Família. Polít Soc. 2011;10(19):97-114.

21. Lima CA, Oliveira APS, Macedo BF, Dias OV, Costa SM. Relação profissional-usuário de saúde da família: perspectiva da bioética contratualista. Rev Bioét. 2014;22(1):15260 .
22. Oliveira MAC. (Re)significando os projetos cuidativos da enfermagem à luz das necessidades em saúde da população. Rev Bras Enferm. 2012;65(3):401-5.

23. Macedo LM, Martin STF. Interdependência entre os níveis de atenção do Sistema Único de Saúde (SUS): significado de integralidade apresentado por trabalhadores da atenção primária. Interface. 2014;18(51):647-59.

24. Souza CR, Botazzo C. Construção social da demanda em saúde. Physis. 2013;23(2) :393413.

Correspondência para:

Hellaynne Cláudia Heiko Yoshi Okuyama e-mail: hellaynneyok@hotmail.com Avenida Campinas, 259, Bairro Banzato 17515-180 - Marília /SP 